\title{
Features of development and using interuniversity scientific and educational network «SYNERGY» for training of engineers in the field of automation, robotics and mechatronic systems
}

\author{
A.V. Bobryakov ${ }^{1}$, V.F. Filaretov ${ }^{2}$, V.G. Homchenko ${ }^{3}$, A.A. Kabanov ${ }^{4}$, B. Katalinic ${ }^{5}$, \\ V.E. Pryanichnikov ${ }^{6}$, S.M. Stazhkov ${ }^{7}$ and A.V. Zuev ${ }^{2}$ \\ ${ }^{1}$ National Research University "Moscow Power Engineering Institute", Moscow; \\ ${ }^{2}$ Far Eastern Federal University and the Institute of Automation and Control Processes FEB RAS, Vladivostok; \\ ${ }^{3}$ Omsk State Technical University, Omsk \\ ${ }^{4}$ Sevastopol National Technical University, Sevastopol; \\ ${ }^{5}$ Vienna University of Technology and DAAAM International, Vienna (Austria); \\ ${ }^{6}$ Institute of Applied Mathematics of M.V. Keldysh RAS, Moscow; \\ ${ }^{7}$ Baltic State Technical University "VOENMEH" of D.F. Ustinov, St. Petersburg;
}

\section{Abstract}

This paper describes a features of development and using of interuniversity scientific and educational network «Synergy» for training of engineers in the field of automation, robotics and mechatronic systems. This network provides: 1) giving of distance lectures by leading professors; 2) carrying out of laboratory classes with remote using laboratory facilities; 3) remote education on project design based on results received during real scientific and commercial projects 4) practices on laboratory and industrial equipment.

Also in paper, some features of software and hardware implementation for remote work with laboratory and industrial equipment in the network is described. Several examples of laboratory work and project design by students remotely implemented using the network is presented.

Keywords: interuniversity research and education network, engineering education, industrial automation, robotics, mechatronics

Received on 30 October 2017, accepted on 12 February, published on 10 July 2018

Copyright (C) 2018 A.V. Bobryakov et al., licensed to EAI. This is an open access article distributed under the terms of the Creative Commons Attribution licence (http://creativecommons.org/licenses/by/3.0/), which permits unlimited use, distribution and reproduction in any medium so long as the original work is properly cited.

doi: 10.4108/eai.10-7-2018.155041

\section{Introduction}

Today, the perspective direction of development of educational technologies is the integration of several universities with industrial companies and scientific organizations by network education [1-4]. But the effectiveness and success of the implementation of networked education depends on many of its features, including breadth and accessibility, as well as the level of direct and real contact of the teacher with the students. Nevertheless, it is already clear that the networked education, having a solid material, technical and scientific basis, is really in demand and will continue to develop. But how this development will work and how effectively it will contribute to the improvement of the level of training of engineering personnel, even if there is a shortage of expensive educational and scientific laboratory equipment and highly qualified teaching staff (especially in peripheral universities), now depends only on initiative groups of universities promoting this form of university education.

In this paper, we describe only some of the most important principles, as well as the features of development and using interuniversity scientific and educational network «SYNERGY» for training of

\footnotetext{
*Email: zuev@dvo.ru
} 
engineers in the field of automation, robotics and mechatronic systems.

Four main components of distance education are realized in the network: 1) reading by leading professors of lecture courses on special subjects for which special educational materials in the form of electronic resources (in several languages), equipped with a large number of demonstration examples and materials; 2) carrying out laboratory works on these disciplines with remote use of laboratory facilities available to all network participants; 3) remote training in project design based on materials already completed for real projects (including for industrial enterprises); 4) operational practices using laboratory and industrial equipment available in the universities of the network.

It is impossible to reflect all four components of the network of education in one article with a sufficient degree of detail. Therefore, below, we will outline the features of the remote implementation of only laboratory practical work and project design.

\section{Features of software and hardware implementation for remote operation with laboratory and industrial equipment in the network}

To realize the possibility of remote work with any mechatronic equipment of various models over the network, specialized software and hardware tools are required that allow remote access via VPN technology. Special software was created based on this technology under the leadership of V. E. Pryanichnikov. This software provides tools to solve synchronization problems, time delays, delineation of access rights and the construction of mesh networks with mobile repeaters that are not sufficiently developed in systems such as ROS [5]. This software implements an intermediate level of "middleware" between the hardware and the application layer, allowing you to merge several mechatronic devices with the ability to remotely access them and providing (if necessary) dynamic configuration changes (autoconfiguration) of system components without stopping devices.

The created software has cross-platform properties (support for x86 \ARM processors and Windows $\backslash$ Linux $\backslash$ Android OS operating systems) for use on various onboard computing devices, is small in size and provides the ability to expand the list of drivers for working with new sensors and drives. When it was created, a Python interpreter was used to provide real-time system operation, and Turing-complete protocols for robot control instead of specialized protocols or graphical interfaces such as GUI MS Robotic Studio, etc.

Python's features allow to implement a system to reprogram the function directly during its operation, which enables the use of supervisory control, when, instead of control commands to the robot actuators are supplied miniprograms of their control. This eliminates the effect of network delays in the formation of robot control, often leading to errors or even emergencies.

For the first time approbation of the created software was carried out with the group control of mobile wheeled and caterpillar robots of the AMUR series (see Figure 1) of the Far Eastern Federal University [6, 7]. Each robot included a personal on-board computer, included in the local computer network; a motion control system with an amplifier of control signals and digital discrete inputs / outputs; a system for measuring distances to detectable objects, built on the basis of ultrasonic sensors and a microcontroller; a video camera for visual monitoring of movements of robots and a video server, included in the local computer network.

The on-board computer of the robot is the ASUS Eee PC 1011CX laptop running under Xubuntu Linux, which receives information from sensors installed on the robot and generates control commands for all of its electronic and mechatronic subsystems. The control software installed on the laptop is launched using supervisord process control tools, which includes two components: a driver that provides an interface for software interaction with the controller via a local area network and specialized software for service functions.

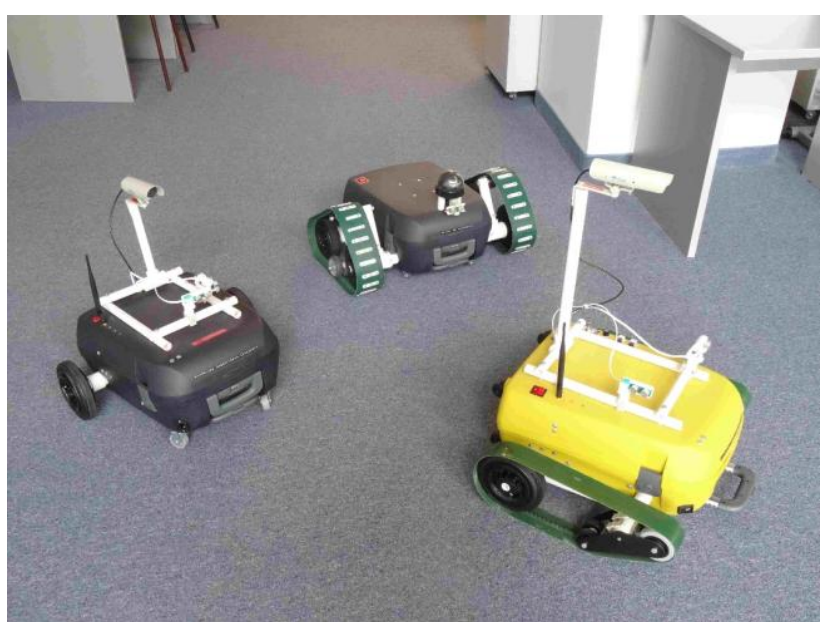

Figure1. Various mobile robots of the AMUR series of Far Eastern Federal University

The robot control system is built on the basis of the microcontroller "Robocon" version $\mathrm{C} 2 \mathrm{~b}$, which transmits information from the odometer sensors of the robot to the on-board computer and generates control signals for all drives in accordance with commands received from the computer. Synthesis of control signals is carried out using a two-channel electronic key operating in PWM mode, the parameters of which are set by the microcontroller. Two ultrasonic sensors of the SRF05 type are used to measure the distances, their signals are processed by the Arduino UNO microcontroller, which in turn is connected to the on-board computer. This microcontroller has more than eight digital inputs and outputs, which allows to expand the composition of sensors and controls. On-board video system of robots uses color analog video cameras, signals of which are digitized with the help of Axis M7001 video 
server, providing simultaneous compression of the received image with the chosen algorithm with the framerate of $50 \mathrm{~Hz}$.

The described software and hardware are used for remote implementation of laboratory practical work by students in several disciplines of specialties related to robotics, production automation and mechatronics. In the conditions of shortage of expensive modern equipment, this expands the range of remote users (students, undergraduates and graduate students) who perform real design in the framework of specific scientific and technical projects. Only work on specific technical projects using real educational and industrial equipment is able to form for most talented students the appropriate level of not only theoretical knowledge and some practical skills, but also relevant competencies in these specialties that will undoubtedly help them successfully solve the tasks of accelerated, purposeful and qualitative modernization of production processes using the latest world achievements of science and technology.

\section{Examples of laboratory practical work and project design of students remotely implemented using a network Group management of mobile robots}

Currently, the important direction of development of mobile robotic systems is the implementation of their group activities in the process of performing complex missions. Group control of wheeled and caterpillar robots of the "AMUR" type with the help of a network when they are moved in different orders (see Figures 1 and 2) is performed by students in laboratory works. The purpose of the laboratory workshop is to obtain theoretical knowledge and practical skills in implementing complex control systems (CS). When preparing and performing laboratory work, students study the composition of the equipment (see Figure 3) of mobile robots (MR), the structure of their CS, the features of the formation and exchange of data between all robots while moving in different orders.

The CS is built on the basis of the master-slave strategy. A leader is appointed in the group. Only leader has full information about the given trajectory of motion. The leader scans the surrounding area with his sensors, revealing obstacles in the path of the group, and the remaining MR form their trajectories based on information about the current position of the leader (see Figure 4).

Students check the efficiency and analyse the advantages of the approach used in the organization of group control in the conditions of limited bandwidth of communication channels between all MR of group, study the features of broadcasting messages from the robot leader to the robots-slaves (this eliminates the multiple transmission of data from the leader to each slave or between slaves) and evaluate the effectiveness of the systems used in comparison with the known ones. Then students study and implement the system's capabilities to change the leader and change the structure in the process of the group's movement.

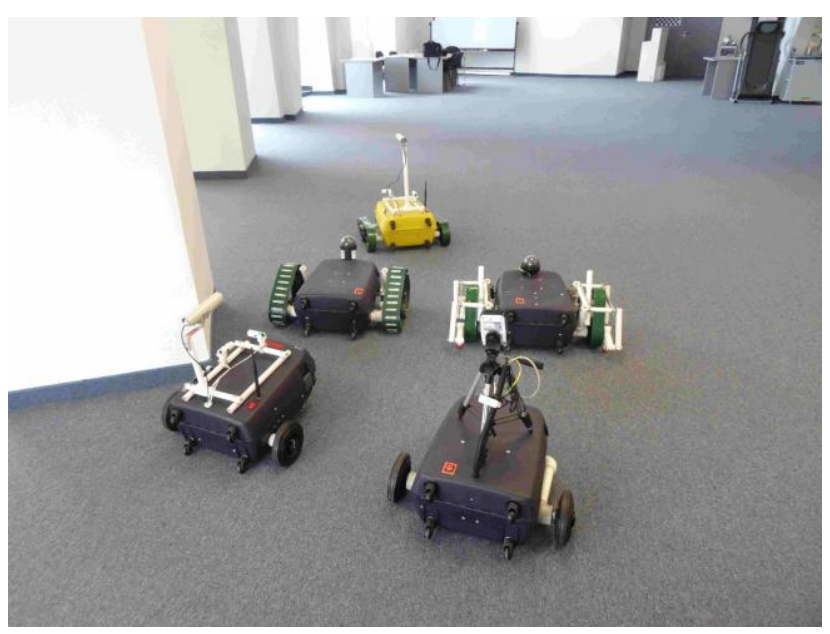

Figure 2. The order of the five mobile robots

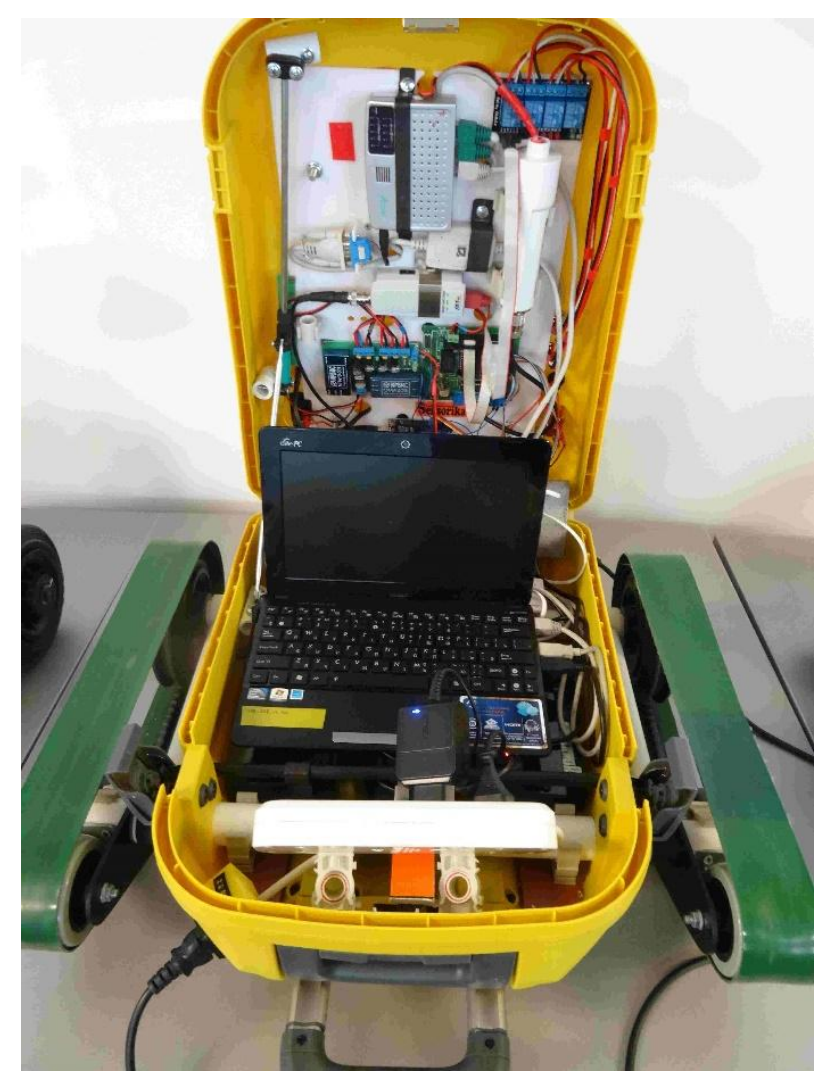

Figure 3. Contents of mobile robots

After conducting a laboratory workshop (including remotely using a network) and having received individual assignments, during the execution of course projects on the discipline "Automated Information and Control Systems", students consistently develop: the architecture 
of a distributed CS providing interaction with local CS of individual MR groups; create the necessary communication components that allow data transfer between individual MR groups; build, and then implement control algorithms of MR depending on the mission.

The final basic task for the ongoing course project is to demonstrate the coordinated movement of the MR group along a given trajectory in a given order (see Figure 4). This demonstration is also carried out over the network, if the course project is carried out by students from different universities.

The course project on group control of MR can be complicated if it is performed by several students. In this case, the leader is additionally given the task of circumventing unexpectedly appeared unknown obstacles in advance. The problem of avoiding obstacles is considered below.

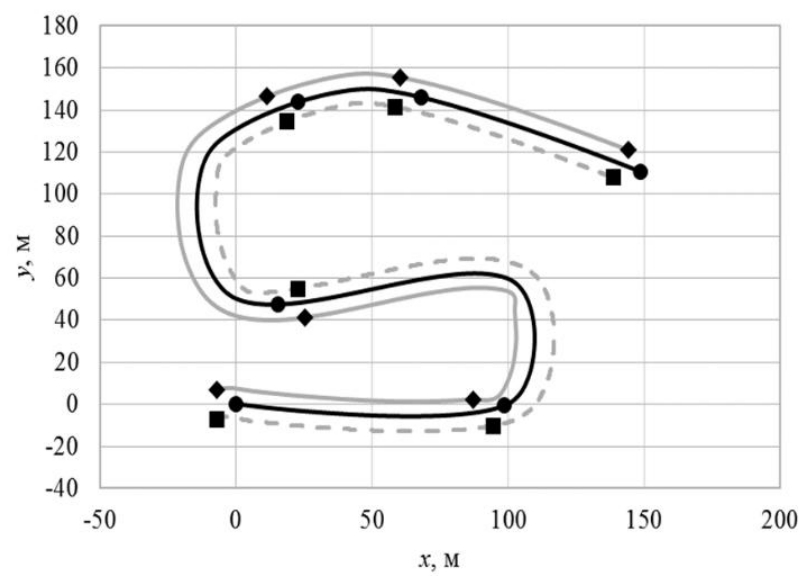

Figure 4. The resulting trajectory of the movement of three robots within the group

Control of autonomous mobile robots in an environment with obstacles. Expanding the scope of MR requires increasing their autonomy when moving in a non-stationary (changing) workspace with previously unknown obstacles. The main source of information about the presence of obstacles near the MR are their onboard range finders. The purpose of laboratory work and subsequent course projects is to provide students with the skills to create CS of MR containing elements of artificial intelligence and ensuring their safe movement in a previously unknown environment (bypassing obstacles). Two approaches to the solution of this problem are considered in the laboratory work. The first is traditional, based on the use of the method of potential fields. To implement this approach, students create the CS architecture that makes it possible to use and configure functions that describe potential fields, as well as eliminating the appearance of effects of falling into the local minimum of the potential field. The second approach consists in the formation of smooth trajectories of MR movement and their adjustment based on the data coming from the onboard range finders. In implementing this approach, students develop a module for the generation of software signals for the movement of MRs, which ensure their motion along trajectories described by Bézier splines of the second order. The MR motion along these trajectories occur at a predetermined speed (perhaps variable).

An example of movement of MR Robotino in an environment with obstacles is shown in Figure 5

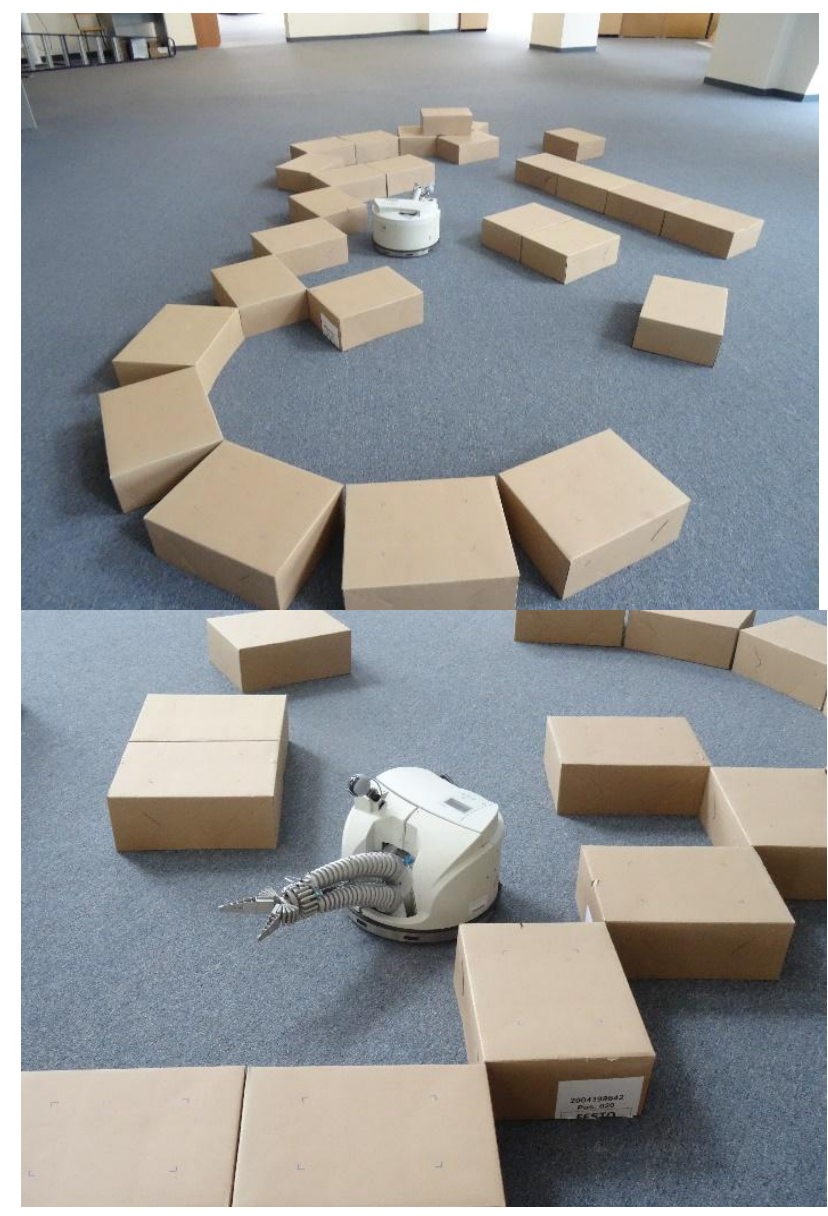

Figure 5. Motion of Robotino robot in an environment with obstacles

Before performing the work, students study the theory and publications prepared by the team of the Department of Automation and Control of the FEFU in the process of real scientific projects in Russian and English [8-10].

The fulfilment of technological operations with the use of vision systems in the presence of unknown deformations in the process of fixing the workpiece before machining. During the implementation of largescale scientific projects with industrial companies of Primorsky Region, a new technology of precise processing of flexible products of arbitrary geometric shape was created with the help of multi-link manipulators (MM) equipped with vision systems (VS) [11-15]. This technology involves a simple and fast fixation of the part in a universal tooling with the possible arbitrary deformation of its geometric shape; scanning of 
a fixed part with the aid of a VS; combining the reference CAD model of the part with its model obtained after scanning to accurately determine machining locations; automatic construction of the trajectories of the working tool of MM.

The purpose of this laboratory work is the development by students of the newly created intellectual robotic technology of accurate and rapid processing of complex parts. At carrying out of laboratory work students at first arbitrarily deform and arbitrarily fix the given out component. Then, it is scanned with a Gocator $3280 \mathrm{~A}$ laser scanner and a computer model of the component is obtained in the form of point clouds (see Figure 6). With the help of the created software, the cloud is compacted, removing the gaps, and combining the CAD model with the obtained three-dimensional model of the scanned randomly deformed component. After that, according to a special algorithm, non-deformed and deformed parts of the component are determined and the points of the workpiece trajectories are transferred from the CAD model to the three-dimensional model of the deformed component. In conclusion, the precise handling of the working tool by the Mitsubishi RV-2FB-D manipulator along the component surface simulating its processing is performed (and observed by students) (see Figure 7).

In more detail, all calculations with a step-by-step simulation of all the above processes are performed by students in design course within the discipline "Robots and their control systems". When a group project is performed with a demonstration of the results obtained in the design assignment, the most capable students are added a section on setting the maximum possible speed for moving the working tool within the specified dynamic control accuracy $[16,17]$.

Controlling the robot with excessive degrees of mobility when processing large-sized products. In the process of continuous execution with the help of MM of various technological operations, there are often situations when some of their mobility degrees go to constraints or there is ambiguity in solving the inverse kinematics problem that determines the law of motion of all degrees of mobility of the multiple links. As a result, during the operation of the MM, unexpected reversals of some of its drives may appear, often leading to emergencies or, at best, to the appearance of a spoilage.

To eliminate this phenomenon, excessive linear degrees of mobility of MM can be used, which allow them not only to process extended objects, but also to choose such locations in space in which unexpected reversals of drives do not occur. The scheme of the laboratory installation that implements the use of additional degrees of mobility of MM is shown in Figure 8. This system consists of MM KUKA 1 and conveyor 2, imitating its additional linear degree of freedom.

When performing laboratory work, students make up a mathematical model of the entire system, select the program trajectory of the working tool of MM relative to the workpiece and perform mathematical modelling of the machining of this part to determine the sections of the trajectory on which unpredictable reversals of electric drives may appear. The part and the program path of the motion are preset in advance such that these reversals necessarily appear. After a special analysis, students determine the required position of the base of the manipulator relative to the detail at which these reversals are excluded.
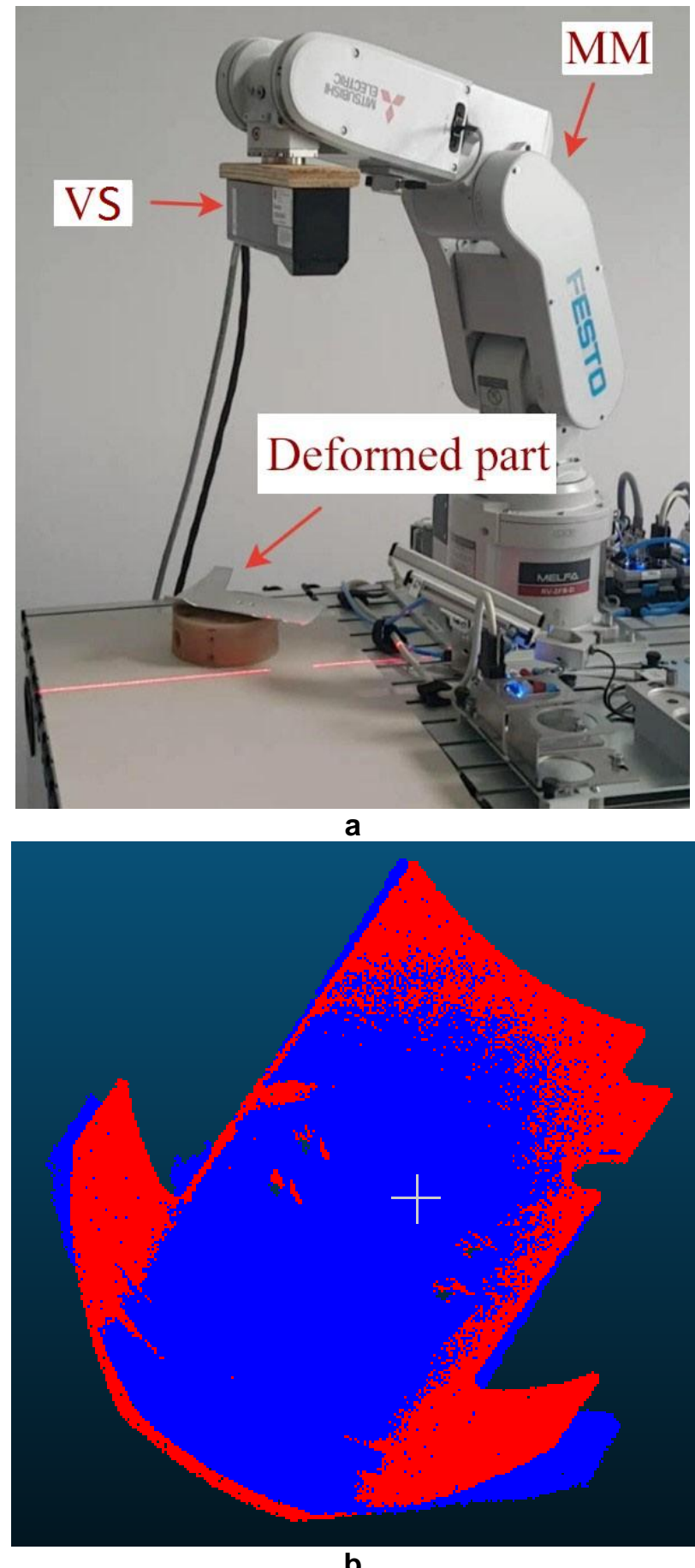

Figure 6. Illustration of scanning an arbitrarily deformed part using VS (a) and obtaining computer models (b) in the form of reference cloud (red cloud) and deformed (blue cloud) part 
Then the base of the MM moves to this preset position. After students in the simulation are convinced of the correctness of the chosen new position, full-scale tests are carried out when the MM of the two indicated motions is performed on the installation shown in Fig.8. In this case, the linear mobility of the MM, simulating the movement of its base relative to the workpiece, is provided by the conveyor.

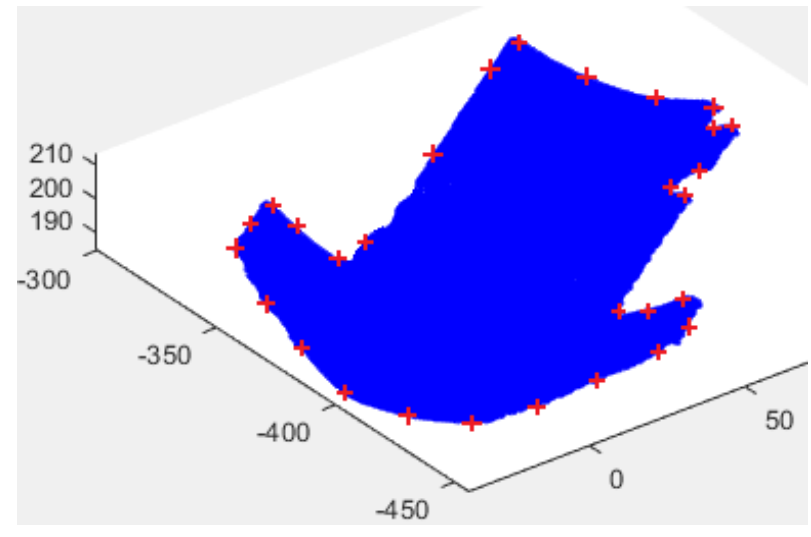

a

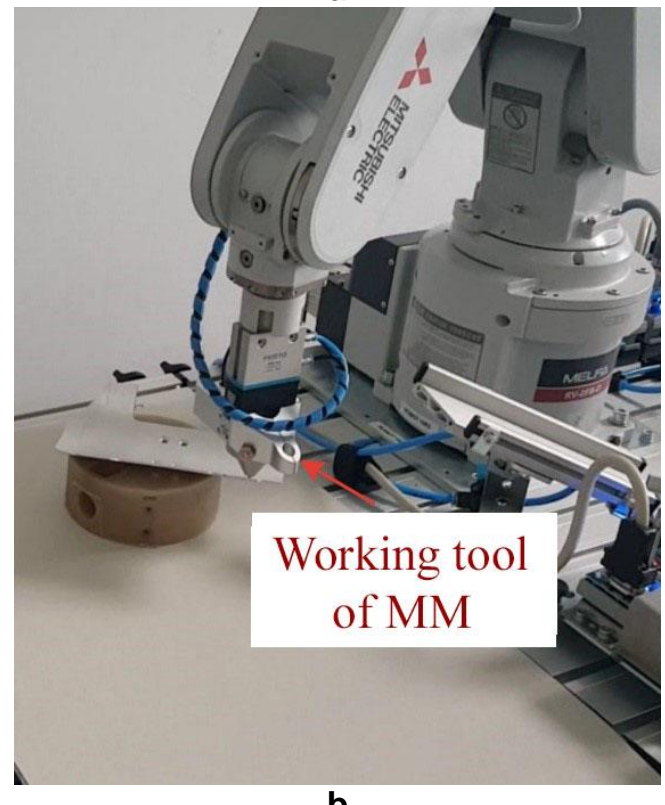

Figure 7. An illustration of the procedures for transferring processing points (red crosses) from the

CAD model to the model of the deformed part obtained during scanning (a) and the realization of the trajectory of processing by the working tool of MM (b)

In the course projects, students solve the problem of performing work operations with the help of MM, when the processing places of products are unknown in advance, and are formed with the help of VS only in the process of performing specific technological operations. At the same time, there is no possibility of preliminary determination of processing areas where unplanned reversals may occur.

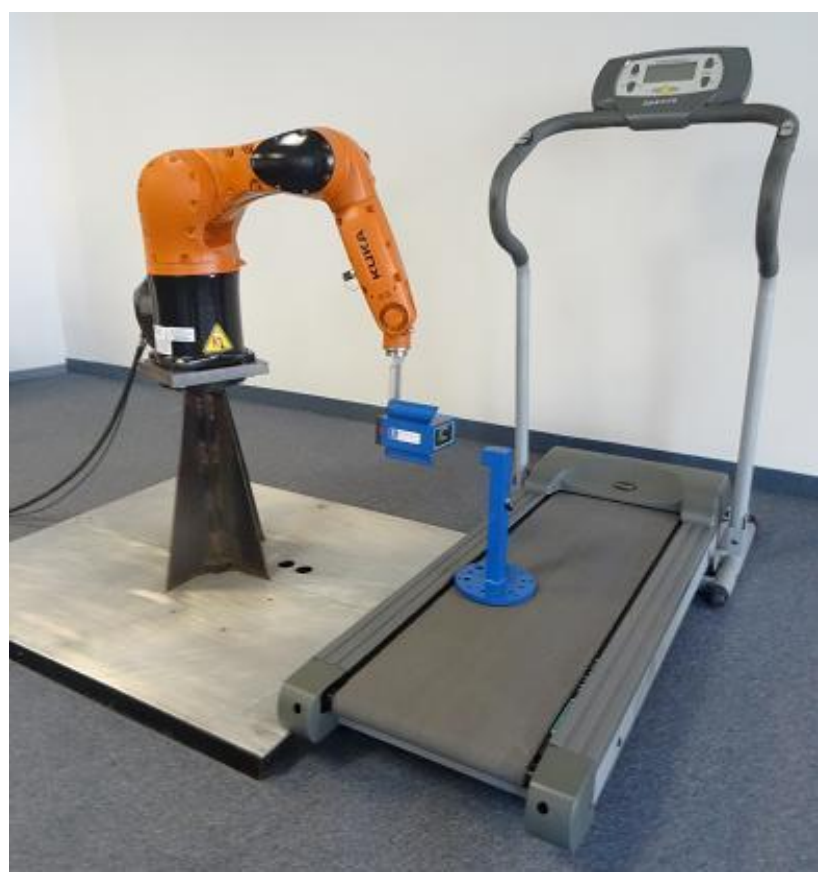

Figure 8. Laboratory installation that creates additional degree of freedom of $\mathrm{MM}$

Therefore, the CS synthesized by the students contains an additional block that automatically moves the base of the $\mathrm{MM}$ in the event of dangerous sections of the continuously formed trajectory of the product are detected. After mathematical modeling of the considered situations, students also study them with the help of the natural system (see Figure 8). All examined and other laboratory installations are connected to the network. This allows to perform laboratory practice remotely.

But by carrying out a laboratory workshop and implementing practical parts of course projects, the possibilities of the network are not exhausted. The most capable students directly participate in grants and projects, implementing new systems protected by patents for inventions and certificates for software products. Only this ultimately contributes to the acquisition of competencies in the design and implementation of intelligent information and control systems of MM with VS at various production facilities.

However, the most valuable feature of the network is that with its help, real developers and designers of various mechatronic systems can present to listeners who are in different regions of the world the most important features and stages of creating these systems. They may share the considerations, methods and approaches that they used to solve unexpectedly emerging problems and challenges. And students can ask questions of interest to them and get answers from immediate executors of successfully implemented projects. This is an invaluable form of getting students the necessary experience and competencies that cannot be found in any published textbooks and teaching aids. 


\section{Conclusion}

Taking into account the limitation of the volume of the material presented, only some features of development and using interuniversity scientific and educational network «SYNERGY» are presented in this article. Despite the already accumulated experience of successful operation of the network in several Russian and International universities, as well as in academic institutions and in industry, this kind of training is only developing. Undoubtedly, in the future there will be new network forms of knowledge transfer and the formation of the required skills and competencies for students. But the main success of networked education will be provided only if, having received the necessary experience, skills and competencies to work with real equipment, students will stop being afraid of new, at first glance, complex equipment and begin to introduce advanced technologies not only on various companies, but also in all spheres of human activity.

\section{Acknowledgements.}

The work is supported by grants from the RFBR (16-29-04195, 16-07-00300).

\section{References}

[1] Shestack V.P. ; Vesna E.B. ; Platonov V.N. (2013) Network education: the best domestic and foreign practices. Contemporary problems of science and education. No. 6.

[2] Zajakina R.A. (2012) Innovative University as a subject of networking // Higher education in Russia. No. 4.

[3] Marković M. ; Kovačević I.K. ; Nikolić O.; and Nikolić B. (2014) INSOS — educational system for teaching intelligent systems. Computer Applications in Engineering Education. Vol. 23. №2. P.268-276.

[4] Chan K.C.; Haythorne S.(2017) A networking laboratory using an integrated mixture of physical equipment and simulators. Comput Appl Eng Educ. Vol. 25. P. 681-692.

[5] Pryanichnikov, V.E.; Bielich, T.; Vitsa, D.; Katalinich, B.; Kirsanov, K.B.; Jars, S.V.; Marzanov, Y.S.; Poduraev, Yu.V.; Helemendik, R.V.; Prysev, E.A.; Ugleshik, A. and Harin, K.V. (2015) Development of educational technology and the associated laboratory-robotarium. Information-measuring and operating systems. Vol.13. P.7-25. (in Russian).

[6] Pryanichnikov, V.E. and Filaretov, V.F. (2015) Autonomous Mobile University Robot AMUR: Technology and Application to Extreme Robotics. Procedia Engineering. Vol.100. P. 269-277.

[7] Pryanichnikov, V.E.; Andreev, V.P. and Prysev, E.A. (2010) Group control of mobile robots, based on the nettechnologies. Proc. of the Int. Conference on Robotics for Security \& Space (Planet \& Earth rovers). St. Peterburg: Poliectnika service. P. 279-283.

[8] Filaretov, V. and Yukhimets, D. (2016) The method of formation of AUV smooth trajectory in unknown environment. Proc. Of Int. Conf. OCEANS'2016. Shanghai, China. P. 1-8.
[9] Filaretov, V.; Yukhimets, D. and Novitsky, A. (2016) The Mobile Robot Navigation Method Based on Onboard Sensors and Camera Data Fusion. Pros. of the Int. Conf.on Computer Graphics, Visualization, Computer Vision and Image Processing. Madeira, Portugal. P. 179-186.

[10] Yukhimets, D. (2017) Neuron Net for Forming Optimal Smooth Trajectories Based on Bezier Splines. Applied Mechanics and Materials. Vol. 865. P. 442-449.

[11] Filaretov, V.F.; Yukhimets, D.A.; Zuev, A.V.; Gubankov, A.S. and Mursalimov, E.Sh. (2016) Development of the method of combining of three-dimensional models of machined parts with their CAD models in the presence of deformations. Problems of Mechanical Engineering and Automation. Vol. 3. P. 60-69. (in Russian).

[12] Filaretov, V.F.; Yukhimets, D.A.; Zuev, A.V. and Gubankov, A.S. (2016) The method of automatic processing of flexible parts of complex spatial form on the basis of information obtained from vision systems and their CAD models. Automation in industry. Vol. 12. P. 5358. (in Russian).

[13] Filaretov, V.; Zuev, A.; Yukhimets, D.; Gubankov, A. and Mursalimov, E. (2016) Method of combination of threedimensional models of details with their CAD-models at the presence of deformations. Proc. of 12th IEEE Conf. on Automation Science and Engineering. Fort Worth, Texas, USA. P. 257-261.

[14] Filaretov, V.; Zuev, A.; Gubankov, A. and Yukhimets, D. (2016) A new approach to automatization of non-rigid parts machining at their deformation by using multilink manipulators with vision system. Proc. of the 2016 IEEE Intern. Conf. on Computer, Control, Informatics and its Applications. Jakarta, Indonesia. P. 227-232.

[15] Filaretov, V.; Yukhimets, D.; Zuev, A.; Gubankov, A. and Mursalimov, E. (2015) The Automatization Method of Processing of Flexible Parts without their Rigid Fixation. Procedia Engineering. Vol. 100. P. 4-13.

[16] Filaretov, V.F.; Gubankov, A.S. and Gornostaev, I.V. (2016) The Formation of Motion Laws for Mechatronics Objects Along the Paths with the Desired Speed. Proc. of International Conference on Computer, Control, Informatics and Its Applications. Jakarta, Indonesia. P. 9396.

[17] Filaretov V.F., Gubankov A.S. Adaptive system forming extremely high speed of multilink manipulator gripper. Proc. of 23rd International DAAAM Symposium on Intelligent Manufacturing and Automation. Zadar, Croatia. Vol. 1. P. 473-476. 